\title{
Fast Recursive Implementation of the Gaussian Filter
}

\author{
D.Demigny, L. Kessal, J. Pons \\ ETIS, UPRESA CNRS 8051, ENSEA et Université de Cergy Pontoise, ENSEA, 6 av. du Ponceau, \\ 95014 Cergy Pontoise Cedex, demigny@ensea.fr
}

\begin{abstract}
On one hand, the convolution with the truncated impulse response (IR) of Gaussian filters leads to a high computation cost when the standard deviation $\sigma$ of the Gaussian increases. On the other hand, recursive filters that approximate the Gaussian filters reduce the computation cost but can only be applied to finite length signals due to the infinity of the IR on both sides.

In this paper, we present a new filter: PAOG. Its IR is a polynomial which is an accurate approximation of the truncated Gaussian IR. Then, we derive a simple and fast recursive implementation of the PAOG filter. The computation cost is independent on $\sigma$. PAOG is particularly suitable for very high speed hardware architecture because the recursive part of the filter uses only adders (without multiplier) which can be implemented in carry-save. This filter can be used with signal of infinite duration (real time). Simultaneously, the first and second derivative of the Gaussian filter are computed without any extra computation cost.
\end{abstract}

Key words: Gaussian filter, recursive filter, polynomial filter

\section{INTRODUCTION}

Gaussian filters and their first and second derivative are often used in image processing for example for denoising and edge detection. The 2D Gaussian filter is the only symmetric filter which is separable. This property reduces the computations to two 1D Gaussian filters used vertically and horizontally. Then our study will use 1D filters without lack of generality.

In this section, we will examine first the finite impulse response (IR) approximations. Then we will discuss some aspects of recursive implementations. Finally we will introduce the PAOG (Polynomial 
Approximation Of Gaussian) filter. The second section will discuss the polynomial approximation and its accuracy. The third section will present the recursive implementation of PAOG and its computation cost. The fourth section will be about hardware implementation.

\subsection{Finite IR approximation of the Gaussian filter}

Usually, the Gaussian infinite IR is truncated to the symmetric $2 \mathrm{M}+1$ center terms. This leads to a convolution scheme for the filter computation. Traditionally, a good approximation requires that $M>3 \sigma$. Then the computation cost and the memory bandwidth increase as $\sigma$. The number of multiplications and additions is more than $(3 \sigma \times, 6 \sigma+)$.

Canny in $[1,2]$ has suggested to use a binomial approximation. The corresponding filter, defined by its $\mathrm{z}$ transform: $H(z)=\left(1+z^{-1}\right)^{n}$, is computed with $n$ iterations of the simple equation $y_{i}=x_{i}+x_{i-1}$ where $y$ and $x$ are the output and the input of the elementary filter. Only $n$ additions are needed. But $\sigma$ increases as $\sqrt{n} / 3$. So the computation cost is: $\left(9 \sigma^{2}+\right)$ which increases very fast with $\sigma$. This approximation is also not accurate for small value of $n$.

\subsection{Infinite IR approximation of the Gaussian filter}

Because the Gaussian has an IR that extends to infinity in both sides, the recursive implementation must employ two recursive filters moving in opposite directions (stability constraint). Each of them has an IR which is approximately a half Gaussian. In [2], Canny has suggested to approximate the half Gaussian by a damped exponential cosine, which leads to a $\mathrm{z}$ transform of the filter with two poles and two zeros. Canny used two passes with the same filter to obtain a good approximation of the Gaussian. The computation cost is then $(12 x, 12+)$. The major advantage of the recursive implementations is that the computation cost becomes independent on $\sigma$. Canny said that this implementation has the lowest cost and must be employed in the future. We will see in this paper that the PAOG filter has a lower computation cost and offers the main advantage to require a move in only one direction which permits its use with real time signals.

\subsection{The PAOG filter}

Canny has defined three criteria to derive the equation of an optimal filter for edge detection in the case of continuous signal. In our previous work $[3,4,5]$, we extend the Canny criteria to sampled signals. Recently, we proved that the optimal Canny first derivative filter can be approximated by 
a simple polynomial IR filter. Because Canny has suggested that its filter can be approximated by the first derivative of Gaussian filter, it means that the integral of our polynomial filter should be a good approximation of the Gaussian.

The PAOG IR has a symmetric shape. Its half IR is a polynomial of degree equal to 4 inside the window size (finite IR). Then, the $\mathrm{z}$ transform of PAOG can be express with a recursive expression. The five poles of this filter are equal to 1 (polynomial property). This particularity permits the computation with only one direction move. Then, the recursive implementation of PAOG is usuable for real time signals. Because the IR is finite, each pole is compensated by a zero of the same value. Details of the PAOG implementation are given in section 3 . We will show that the computation cost is only $(4 \times, 11+)$. In the next section, we will discuss the quality of the Gaussian approximation with the PAOG filter.

\section{POLYNOMIAL APPROXIMATION}

\subsection{The PAOG impulse response}

The size of the IR is defined as $2 \mathrm{w}+1$. The expression of the IR $h^{w}$ of the PAOG filter is:

$$
h_{k}^{w}=C_{p} \cdot(w+2-|k|)(w+1-|k|)\left(-3 k^{2}+(2 w+3)|k|+w(w+3)\right)
$$

where $C_{p}$ is a normalization constant chosen to obtain a sum of the filter coefficients equal to one. This choice sets the amplification to one for low frequency signals independently of $w$.

$$
C_{p}=\frac{5}{2 w(w+1)(w+2)(w+3)(2 w+3)}
$$

The Gaussian IR is:

$$
g^{\sigma}=C_{g} e^{-\frac{k^{2}}{2 \sigma^{2}}}
$$

For each value of $w$, it exists a value of $\sigma$ for which $h^{w}$ will be an accurate approximation of the Gaussian IR $\mathrm{g}^{\sigma}$ as explained below. Using the minimization of the absolute quadratic error, we find: 


$$
\sigma=0.3217 w+0.481
$$

On figure 1, we show the half positive IR $h^{w}$ and $g^{\sigma}$ for $w=20$ and $\sigma=6.915$. We see that the largest difference is near the extremity of the window, i.e. for the lowest values of the response.

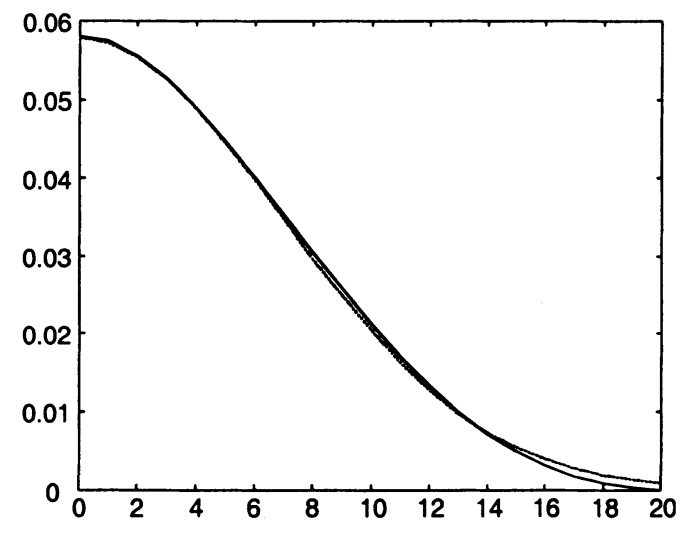

Figure 1. Half impulse responses of the Gaussian filter (dashed) with $\sigma=6.915$ and of the PAOG filter (solid) with $\mathrm{w}=20$

\subsection{Extension to different $\sigma$ values}

Usually, for image processing, it is not necessary to use accurate values of $\sigma$. Then the fact that only a finite number of $\sigma$ values is available since $w$ is an integer is not a drawback. However, it is possible to obtain different $\sigma$ values if a cascade of PAOG filters is used. For a cascade of $n$ filters, if the filter $i$ corresponds to $\sigma_{\mathrm{i}}$, the global $\sigma$ is defined as:

$$
\sigma^{2}=\sum_{i=1}^{n} \sigma_{i}^{2}
$$

\subsection{Accuracy of the Gaussian approximation}

We note $g^{w}{ }_{\sigma}$ the restriction of the function $g_{w}$ to the window of size $(2 w+1)$. Three different measures are used to study the error inside the window.

a) The mean of the relative quadratic error: $m r q$

$$
m r q=\frac{1}{2 w+1} \sqrt{\sum_{-w}^{+w}\left(1-\frac{h^{w}}{g_{w}^{\sigma}}\right)^{2}}
$$


b) The mean of the absolute quadratic error normalized by the value of $h^{\mathrm{w}}{ }_{0}$ : maq

$$
m a q=\frac{1}{h_{0}^{w}(2 w+1)} \sqrt{\sum_{-w}^{+w}\left(g_{w}^{\sigma}-h^{w}\right)^{2}}
$$

c) The maximum of the absolute quadratic error normalized by the value of $h^{w}(0): M a q$

$$
M a q=\frac{\max _{k}\left|g_{w}^{\sigma}(k)-h^{w}(k)\right|}{h^{w}(0)}
$$

The figure 2 shows the errors in $\%$ for $w$ between 1 and $20(0.8<\sigma<6.91)$. $m r q$ is less than $6 \%, M a q$ is approximately constant $(2 \%)$ and $m a q$ is less than $0.5 \%$.

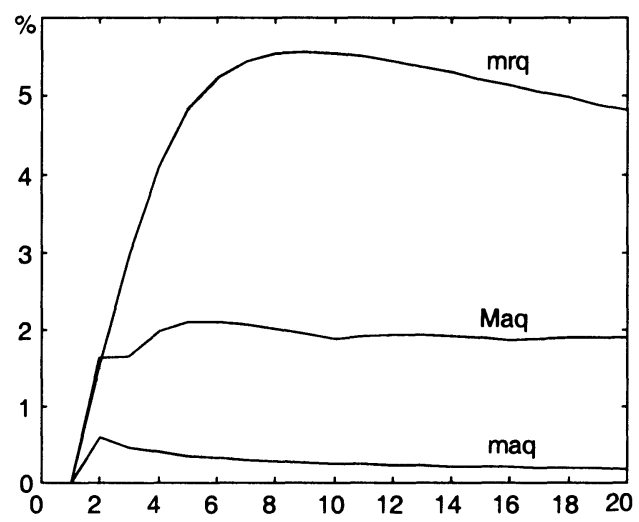

Figure 2. Mean absolute (maq), maximum absolute (Maq) and mean relative (mrq) quadratic error between $\mathrm{g}^{\sigma}$ and $\mathrm{h}^{\mathrm{w}}$ in $\%$

An other measure looks for the truncative effect. The ratio between the sum of the filter coefficients of $g^{\sigma}$ outside the window and the sum of the filter coefficients of $g^{\sigma}$ inside the window is less than $1 \%$ for all the $w$ values. All these results confirm that the PAOG filter is a good approximation of the Gaussian filter. 


\section{RECURSIVE IMPLEMENTATION}

\subsection{Z transform}

The $\mathrm{z}$ transform of $h^{w}$ can be cut in:

$$
H^{w}(z)=\sum_{k=-w}^{0} h_{k}^{w} z^{-k}+\sum_{k=0}^{+w} h_{k}^{w} z^{-k}-h_{0}^{w}
$$

To compute the second sum part of (9) we have first expanded the polynomial (1) as:

$$
\sum_{k=0}^{+w} h_{k}^{w} z^{-k}=\sum_{i=0}^{4} a_{i} \sum_{k=0}^{+w} k^{i} z^{-k}
$$

where $a_{i}$ are functions of $w$. The second sum in (10) can be analytically evaluated:

$$
\sum_{k=0}^{+w} k^{i} z^{-k}=\frac{N_{i}^{w}\left(z^{-1}\right)}{\left(1-z^{-1}\right)^{i}}
$$

where $N_{i}^{w}\left(z^{-1}\right)$ is a polynomial of degree $i$ of the variable $z^{-1}$. Introducing (11) in (10) and using the same method to compute the first sum part of (9), one obtains the expression of $H^{w}(z)$ :

$$
H^{w}(z)=C_{p}^{\prime} \frac{z^{-2}\left(1+z^{-1}\right) N^{w}(z)}{\left(1-z^{-1}\right)^{5}}
$$

with:

$$
\begin{aligned}
N^{w}(z)= & w\left(z^{(w+2)}-z^{-(w+2)}\right)+(w+3)\left(z^{-(w+1)}-z^{(w+1)}\right) \\
& +(2 w+3)\left(z-z^{-1}\right)
\end{aligned}
$$

and:

$$
C_{p}^{\prime}=\frac{30}{w(w+1)(w+2)(w+3)(2 w+3)}
$$




\subsection{Stability}

Because the poles are located on the unit circle, the stability of the filter is obtain if and only if the zeros of the numerator $N^{w}$ compensate exactly the poles. So, any computation error on $N^{w}$ leads to instability ! This prohibits the use of floating point numbers for the filter computation because summation of two numbers very different in value leads to an approximated result. The computations must be made with integer numbers. The input data and the filter coefficients are integer numbers too. The normalization by the constant $C_{p}^{\prime}$ must be made at the end of the computation and, in this case, the floating point notation is authorized (after and outside the recursive loop). This is not so restrictive because the input data in image processing are usually represent by integer values. It is also a great advantage for the implementations on the cheaper DSP or on hardware (ASIC or FPGA).

\subsection{Computation algorithm}

We define:

$$
a_{0}=w, \quad a_{1}=w+3, \quad a_{2}=2 w+3
$$

The current data input is $x_{i}$ and one wants to compute the output $y_{i}$. The computation progresses towards increasing values of $i$. All the $t_{j}$ and $n$ are simple temporary variable.

$$
\begin{aligned}
& n=a_{0}\left(x_{i+w+2}-x_{i-w-2}\right)+a_{1}\left(x_{i-w-1}-x_{i+w+1}\right)+a_{2}\left(x_{i+1}-x_{i-1}\right) \\
& t_{1}=t_{1}+n \\
& t_{2}=t_{2}+t_{1} \\
& t_{3}=t_{3}+t_{2} \\
& t_{4}=t_{4}+t_{3} \\
& t_{5}=t_{5}+t_{4} \\
& y_{i}=C_{p}^{\prime}\left(t_{6}+t_{5}\right) \\
& t_{6}=t_{5}
\end{aligned}
$$

\subsection{Initialization of the algorithm}

For image processing, because the number of data on each line (or each column) is finite, attention can be made for the initialization to avoid 
transient response near each side. More important, because five integrations are applied on the signal, bad initialization leads to overflow and instability !

To avoid instability and transient responses, we proceed as follow: near the left side (or at reset for real time signals), to avoid transient response near $x_{0}$, one supposes that $x_{0}$ is a continuous value for negative $i$ indexes. $t_{1 . .4}$ and $t_{6}$ are zeroed and $t_{5}$ is initialized to $2 x_{0} / C_{p}^{\prime}$ which is always an integer when $x_{0}$ is an integer. At iteration $i$, unavailable input data $x_{i+j}$ are replaced in (16) with $x_{0}$. Input data $x_{i+j}$ are replaced by their right values as soon as they become available. For the right side (image processing), if $N$ is the index of the last pixel, the data $\left(x_{i+w+2}, x_{i+w+1}, x_{i+1}\right)$ can be replaced by $x_{N}$ when they become unavailable.

\subsection{Performances of the programming version of PAOG}

The PACG algorithm uses only 11 additions and 4 multiplications. An other advantage is the reduced memory bandwidth needed. Only 5 memory accesses by pixel computed are necessary. To compare the performances, we have implemented the Gaussian filter with a simple convolution and the PAOG filter for the same window size $w$. We have also tested the recursive solution proposed by Canny, see $\$ 1.2$. Programs are written in C language on a PC, and integer numbers are used. The PAOG filter is 1.37 times faster than the Canny recursive filter. Both have a computation time independent on $\sigma$. The table I shows the speed gain of the PAOG filter compared to the convolution as a function of $w$.

\begin{tabular}{lllllll}
\hline$w$ & 2 & 6 & 10 & 14 & 18 & 20 \\
\hline gain & 1.77 & 4.25 & 6.71 & 9.17 & 11.6 & 12.9 \\
\hline
\end{tabular}

Table 1. Speed improvement

The gain increases as $w$ or $\sigma$. The superiority of the recursive scheme of PAOG on the convolution scheme is clear.

\section{HARDWARE IMPLEMENTATION}

\subsection{Functional description}

The figure 3 shows the main details of the hardware implementation. Delayed data are obtained at the output of the registers and of the two memories A and B used as FIFO of size $w$. The time consuming operators are essentially the multipliers. Because they are only used in the no recursive part, retiming and pipelining can be easily employed to increase the speed. 
The recursive part uses only adders. So, this part can be easily accelerated with carry-save or bit pipelined adders. Looking to figure 3, it will be remarked that in the same time, the filter computes the fourth first derivatives $\left(y^{(1)}\right.$ to $\left.y^{(4)}\right)$ of the output without extra computation !

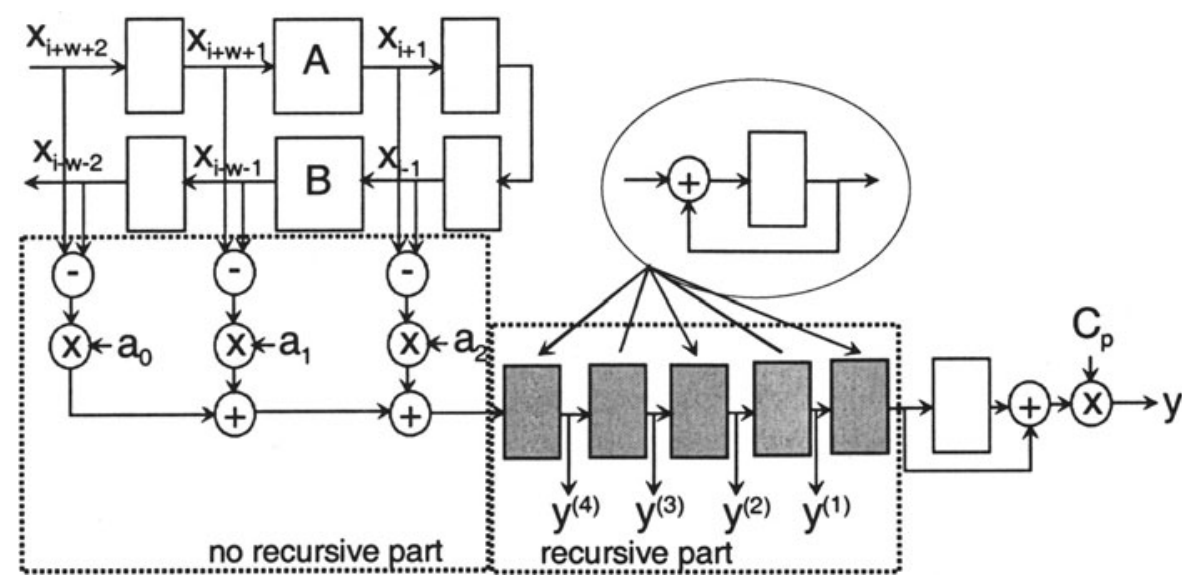

Figure 3. Hardware design. Pipelined registers are not detailed on this figure

\subsection{Sizing of data and computations}

The presence of the integrators in the recursive loop complicates the operators and registers sizing. To ensure the filter stability, one must avoid the saturation of the computations. One supposes that input data are $M$ bits unsigned numbers. Multipliers have low size because $M$ is about 8 bits in image processing and the highest coefficient is $2 w+3$. If this coefficient is coded with 8 bits, $w=126$ and the impulse response is extend to 253 pixels !

The output of the no recursive part is a signed integer. It is coded with:

$$
M+3+\log _{2}\left(w+\frac{3}{2}\right) \quad \text { bits }
$$

It is impossible to size an integrator with a reasoning starting from input and progressing towards output. One must proceed from output towards input. Because the global amplification is one, the $y$ result on figure 3 as an integer part coded with $M$ bits. The output size of the last adder is unsigned and coded with:

$$
M-\log _{2} C_{p}^{\prime} \quad \text { bits }
$$


Because the static amplification of the last adder is 2, the output of the last integrator uses one bit less than (18). An integrator with input $u$ and output $v$ computes:

$$
v_{n}=u_{n}+v_{n-1}
$$

Inverting this equation leads to:

$$
u_{n}=v_{n}-v_{n-1}
$$

The input of an integrator uses one more bit than its output (worst case). Then, it is possible to compute an over-estimation of the $y^{(4)}$ register size (figure 3) which is an unsigned integer:

$$
M+3-\log _{2} C_{p}^{\prime} \approx M-1+5 \log _{2}(w+3)
$$

The other operators and registers size can be easily deduced from (21). For a 32 bits DSP implementation and for 8 bits input data, (21) leads to $w_{\max }=29$ or $\sigma_{\max }=9.8$.

An other way to compute the register size uses a property of the addition with two's complement numbers (thanks to J. Liénard which remember me this property). There is no need to take care of the overflow of the partial results in a chain of adders. If $O$ is the number of bits of the output of the last integrator:

$$
O \approx M-5+5 \log _{2}\left(w+\frac{3}{2}\right)
$$

This result is less restrictive than the previous one. The same size $O$ can be used for each integrator. For example, 32 bits computations allow a maximum of $w=47$ for 8 bits input data or $w=17$ for 16 bits input data.

\subsection{FPGA implementation}

We have estimated the maximum sampling data rate for an implementation a Xilinx VIRTEX XC2V1000-5 FPGA. This device includes fast $18 \times 18$ bits multipliers which can compute $8 \times 9$ bits multiplications in less than $3 \mathrm{~ns}$. Registers and adders of our implementation are over-estimated to 32 bits width. We assume that pipelining registers are inserted between each operator of the no-recursive part. In this case, the maximum sampling rate (144 Mhz) is limited by the accumulators (integrators). This sampling rate 
can be double by the use of block adders (each 32 bits adder is cut in four 8 bits adders laterally pipelined).

\section{CONCLUSION}

We have shown that the PAOG filter is a good approximation of the Gaussian filter. It has a finite impulse response, but on the contrary to the conventional approaches (convolution), we have proposed to implement the PAOG filter with a recursive scheme. Usually, recursive filters on image processing require computation moves in both directions (left to right and right to left for horizontal computation). The very unusual choice of a polynomial impulse response leads to a limit case. It derives that only one move is necessary. Then the PAOG filter can be employed with real time data (signal processing) and no frame storage is needed for the vertical computation (image processing). Usually, one thinks that floating point computations are more accurate than fixed point or integer ones. Surprisingly, in the PAOG case, it is the opposite. Only the integer numbers ensure the filter stability! As strange as it is, this simple filter permits very fast computations both in hardware and software.

\section{REFERENCES}

[1] J. Canny, "A computational approch to edge detection", IEEE Transactions on Pattern Analysis And Machine Intelligence, vol. 8, pp. 679 - 714, 1986.

[2 J.F. Canny, "Finding edges and lines in images", Tech. Rep. AI-TR-720, MIT Artificial Intell. lab., 1983.

[3] D. Demigny, and T. Kamleh, “A discrete expression of Canny's criteria for step edge detection performances evaluation”, IEEE Pattern Analysis and Machine Intelligence, vol. 19, $\mathrm{n}^{\circ} 11$, pp. $1199-1211$, november 1997.

[4] D. Demigny, F. Garcia Lorca, and L. Kessal, "Evaluation of edge detector performances with a discrete expression of Canny's criteria", Proc. International Conference on Image Processing, Washington, october 1995, IEEE ICIP, n², pp. $169-172$.

[5] D. Demigny, "Extension of Canny's discrete criteria to second derivative filters: towards a unified approach", Proc. International Conference on Image Processing, Chicago, october 1998, IEEE ICIP. 